\title{
Video Article \\ Evaluation of Keratinocyte Proliferation on Two- and Three-dimensional Type I Collagen Substrates
}

\author{
Hitomi Fujisaki ${ }^{1}$, Sugiko Futaki ${ }^{2}$, Kazunori Mizuno ${ }^{1}$, Shunji Hattori ${ }^{1}$ \\ ${ }^{1}$ Nippi Research Institute of Biomatrix \\ ${ }^{2}$ Department of Anatomy and Cell Biology, Osaka Medical College
}

Correspondence to: Hitomi Fujisaki at fujisaki@nippi-inc.co.jp

URL: https://www.jove.com/video/59339

DOI: doi:10.3791/59339

Keywords: Bioengineering, Issue 146, type I collagen, fibril/non-fibrous form, two-/three-dimensional culture substrates, on-gel culture, keratinocyte culture, proliferation

Date Published: 4/22/2019

Citation: Fujisaki, H., Futaki, S., Mizuno, K., Hattori, S. Evaluation of Keratinocyte Proliferation on Two- and Three-dimensional Type I Collagen Substrates. J. Vis. Exp. (146), e59339, doi:10.3791/59339 (2019).

\section{Abstract}

Type I collagen, useful as a substrate for cell culture, exists in two forms: the two-dimensional, non-fibrous form and three-dimensional, fibril form. Both forms can be prepared with the same type I collagen. In general, the non-fibrous form promotes cell adhesion and proliferation. The fibril form (gels) provides more physiological conditions in many types of cells; therefore, gel culture is useful for examining physiological behaviors of cells, such as drug efficacy.

Researchers can select the appropriate form according to the purpose of its use. For example, in the case of keratinocytes, on-gel culture has been used as a wound healing model. FEPE1L-8, a keratinocyte cell line cultured on the non-fibrous form of type I collagen, promote cell adhesion. Notably, keratinocyte proliferation is slower on the fibril form than the non-fibrous form. Protocols for the preparation of type I collagen for cell culture are simple and have wide applications depending on the experimental needs.

\section{Video Link}

The video component of this article can be found at https://www.jove.com/video/59339/

\section{Introduction}

Interstitial connective tissues comprise a three-dimensional protein meshwork of heterogeneous composition, primarily composed of type I collagen fibrils $s^{1}$. Collagen fibrils play a key role as a scaffold for cells ${ }^{1,2,3}$ and interact with other extracellular matrix (ECM) proteins ${ }^{3}$. In vitro, different forms of type I collagen can be used as substrates for cell culture depending on the handling method ${ }^{1,2,3,4}$. Under acidic conditions, type I collagen maintains the non-fibrous form ${ }^{5}$. Coating the surface of culture dishes with the non-fibrous form promotes cell adhesion and proliferation $^{6,7}$. At physiological $\mathrm{pH}$ and temperature, type I collagen molecules reassemble into fibrils that form gels possessing a threedimensional structure ${ }^{1,2,3,4,5,6,7,8}$. There are several important differences between the fibril and non-fibrous forms of type I collagen, including matrix stiffness and efficiencies of reconstruction of ECM components by the cells during the culture ${ }^{1}$. Matrix stiffness is one of the most studied regulatory factors of cell culture ${ }^{1,9}$. However, the complex interactions between substrates and cells remain to be clarified. To examine the complex interactions between cells and environmental factors, a simple system is useful. Comparison of the cellular behavior on the two different forms of collagen may help to simplify the effect of environmental factors. Depending on the purpose of their use, different forms of type I collagen can be selectively used. Normally, keratinocytes are in contact with the basement membrane but not with type I collagen. However, during wound healing, keratinocytes move to the dermal connective tissue, proliferate, and heal the wound ${ }^{10}$.

Recently, we demonstrated that the concentration of extracellular calcium is important for proliferation of keratinocyte line cells by using the culture system on the fibril form of type I collagen mimicking the dermal connective tissue ${ }^{11}$. When the keratinocyte cell line FEPE1L-8 was cultured on the fibril form of type I collagen, the shape of the cells was round and their proliferations were stopped at an extracellular calcium concentration of $30 \mu \mathrm{M}^{11}$. When the calcium concentration was increased to $1.8 \mathrm{mM}$, cell growth was recovered ${ }^{11}$. The cells grew under both calcium concentrations $(30 \mu \mathrm{M}$ and $1.8 \mathrm{mM})$ when cultured on the non-fibrous form ${ }^{11}$, whereas they were more sensitive to the exogenous calcium concentration when cultured on the fibril form. FEPE1L-8 was generated through transfection with the papillomavirus type 16 transforming genes E6 and E7 from human cervical carcinoma, non-tumorigenic, inhibit unlimited proliferation with limited differentiation potential like normal keratinocytes ${ }^{12,13}$. FEPE1L-8 cells can be maintained by using some kinds of keratinocyte specific medium, including K110 TypeII with additive supplement K-1 (K110) ${ }^{6}$. Here, we describe the culture protocol of the human keratinocyte cell line on the non-fibrous and fibril forms of type I collagen. 


\section{Preparation of keratinocyte culture medium}

NOTE: Perform all procedures under aseptic conditions.

1. Add $5 \mathrm{~mL}$ of penicillin-streptomycin and $10 \mathrm{~mL}$ of additive supplement $\mathrm{K}-1$ to $500 \mathrm{~mL}$ of $\mathrm{K} 110$ Type-II medium (K110) using a pipette.

\section{Preparation of the fibril form of type I collagen}

NOTE: Perform procedures until step 2.6 under aseptic conditions.

1. Keep 10x phosphate-buffered saline (PBS (-)), deionized water, collagen, a 96-well culture plate, and an empty 2-mL tube on ice. NOTE: Do not use the same plate to prepare the fibril and non-fibrous forms.

2. Add $1.12 \mathrm{~mL}$ of deionized water to an empty $2 \mathrm{~mL}$ tube using a pipette. Add $200 \mu \mathrm{L}$ of $10 x$ PBS (-) to the deionized water in the $2 \mathrm{~mL}$ tube using a pipette. Gently shake the tube several times.

3. Add $0.66 \mathrm{~mL}$ of collagen to the $2 \mathrm{~mL}$ tube using a pipette. Gently and quickly shake the tube several times. NOTE: Avoid bubble formation in the solution as much as possible. Prepare collagen solution immediately prior to use.

4. Pour $100 \mu \mathrm{L}$ of the collagen solution from step 2.3 into each well of the 96 -well culture plate. Gently shake the culture plate in a left to right motion.

NOTE: Cover the entire surface of the wells with the collagen solution. If the solution does not cover the entire surface, spread the solution using a pipette tip. Avoid bubble formation in the solution as much as possible.

5. Place the culture plate in a $\mathrm{CO}_{2}$ incubator and incubate at $37^{\circ} \mathrm{C}$ for $1 \mathrm{~h}$. Check the gelation of collagen and move the culture plate to a clean bench.

NOTE: Confirm gelation by tilting the culture plate.

6. Gently pour $150 \mu \mathrm{L}$ of $\mathrm{K} 110$ on the gels using a pipette along the wall of the well, place the culture plate in a $\mathrm{CO}_{2}$ incubator and incubate at 37 ${ }^{\circ} \mathrm{C}$ for $1 \mathrm{~h}$. Move the culture plate to a clean bench. Prior to cell culture, gently discard the K110 using a pipette. NOTE: To protect the gels, the tip of the pipette should touch the wall of the well.

\section{Preparation of the non-fibrous form of type I collagen}

NOTE: Perform all procedures under aseptic conditions.

1. Add $4 \mu \mathrm{L}$ of collagen to $1.2 \mathrm{~mL}$ of $1 \mathrm{mM}$ hydrochloric acid $(\mathrm{HCl})$ in a $1.5 \mathrm{~mL}$ tube and gently mix using a pipette. NOTE: Prepare collagen immediately prior to use. Keep collagen and $\mathrm{HCl}$ chilled until the time of use.

2. Pour $100 \mu \mathrm{L}$ of collagen into each well of a 96 -well culture plate using a pipette. Gently shake the culture plate in a left to right motion and incubate at room temperature for $1 \mathrm{~h}$.

NOTE: Ensure that the entire surface of the wells is covered with solution.

3. After the incubation, discard the collagen solution and wash the wells with PBS (-) twice using a pipette.

4. Pour $150 \mu \mathrm{L}$ of $1 \%$ bovine serum albumin/PBS (-) (1\% BSA) to a well of the 96 -well culture plate and incubate at room temperature for $1 \mathrm{~h}$. Prior to cell culture, discard the $1 \%$ BSA.

NOTE: Ensure that the entire surface of the wells is covered with solution.

\section{Culture of FEPE1L-8 cells}

NOTE: Perform all procedures under aseptic conditions.

1. Maintain FEPE1L-8 cells in $\mathrm{K} 110$ in a $100 \mathrm{~mm}$ culture dish in a $\mathrm{CO}_{2}$ incubator and incubated at $37{ }^{\circ} \mathrm{C}$ and $5 \% \mathrm{CO}_{2}$, to semi-confluency.

2. Prepare $\mathrm{K} 110$, trypsin, and trypsin inhibitor in a water bath at $37^{\circ} \mathrm{C}$.

3. Carefully remove the medium from the culture dish, add $3 \mathrm{~mL}$ of $0.05 \%$ trypsin using a pipette, place the dish in a $\mathrm{CO}_{2}$ incubator and incubate at $37^{\circ} \mathrm{C}$ for $5 \mathrm{~min}$. After incubation, check cell detachment from the surface of the culture dish using phase-contrast microscopy at $10 \mathrm{x}$ magnification.

NOTE: The morphology of the detached cells becomes round. If the cells spread, incubate for an additional period of 5 min.

4. Add $3 \mathrm{~mL}$ of trypsin inhibitor and collect the detached cells in a $15 \mathrm{~mL}$ centrifuge tube using a pipette. Centrifuge the cells in a $15 \mathrm{~mL}$ centrifuge tube at $200 \times \mathrm{g}$ for $5 \mathrm{~min}$.

5. Discard the supernatant and re-suspend the pellet in $10 \mathrm{~mL}$ of $\mathrm{K} 110$ using a pipette. Count the cells using phase-contrast microscopy at $10 \mathrm{x}$ magnification and prepare cell concentration at $5.0 \times 10^{4}$ cells $/ \mathrm{mL}$ by appropriate dilution with $\mathrm{K} 110$.

6. Gently seed $0.1 \mathrm{~mL}$ of $\mathrm{K} 110$ with cells in each well of the culture plate using a pipette along the wall of the well. Place the culture plate in a $\mathrm{CO}_{2}$ incubator and incubate at $37^{\circ} \mathrm{C}$ for the indicated time ( $2 \mathrm{~h}, 1$ day, 3 days).

NOTE: To protect the gels, the tip of the pipette should touch the wall of the well.

\section{Estimation of the number of viable cells}

1. Incubate $\mathrm{K} 110$ in a water bath at $37^{\circ} \mathrm{C}$. Mix $130 \mu \mathrm{L}$ of tetrazolium salt, 2-(2-methoxy-4-nitrophenyl)-3-(4-nitrophenyl)-5-(2,4disulfophenyl)-2H tetrazolium, monosodium salt (WST-8), and $1.3 \mathrm{~mL}$ of $\mathrm{K} 110$ in a 2-mL tube using a pipette. 
2. Move the culture plate to a clean bench and gently discard the K110 using a pipette. Gently wash away non-adherent cells with $\mathrm{K} 110$ by using a pipette

3. Add $110 \mu \mathrm{L}$ of $\mathrm{K} 110$ mixed with WST-8 in each well using a pipette, place the culture plate in a $\mathrm{CO}_{2}$ incubator and incubate at $37{ }^{\circ} \mathrm{C}$ for $2 \mathrm{~h}$.

4. Move the culture plate to a clean bench, collect $100 \mu \mathrm{L}$ of the conditioned medium from each well, and move it into a well of another 96 -well culture plate. Measure absorbance at a wavelength of $450 \mathrm{~nm}\left(\mathrm{OD}_{450}\right)$ using a microplate reader and estimate the number of viable cells.

\section{Representative Results}

A schematic representation of the treatment of the surfaces of culture dishes using type I collagen is depicted in Figure 1. Cell morphologies observed on the non-fibrous and fibril forms are presented in the left- and right-side panels of Figure 2, respectively. FEPE1L-8 cells were cultured for $2 \mathrm{~h}$ (upper panels) and 3 days (lower panels). In the initial $2 \mathrm{~h}$ of culturing, the cells adhered and spread on both forms of collagen (Figure 2, upper panels). Three days after seeding, cells on the non-fibrous form continued to spread and cell numbers increased (Figure 2, lower left panel). In contrast, the cells on the fibril form showed limited spreading (Figure 2, lower right panel). FEPE1L-8 cells continued to proliferate on the non-fibrous form of type I collagen (Figure 3, solid black line, closed black circles) and on the untreated dish surfaces (Figure 3, dotted gray line, closed gray circles). In contrast, cells did not proliferate on the fibril form (Figure 3, dotted line, open circles). Figure 2 and Figure 3 have been modified from Fujisaki et al $^{11}$.

\section{- :type I collagen molecule}

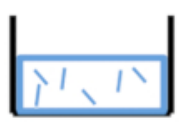

at room temp. in acidic $\mathrm{pH}$

for $1 \mathrm{~h}$

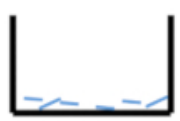

non-fibrous form

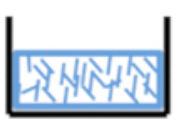

at $37^{\circ} \mathrm{C}$

in neutral $\mathrm{pH}$

for $1 \mathrm{~h}$

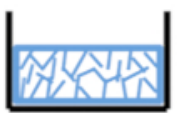

fibrils (gel form)

Figure 1: Schematic representations of culture dish surfaces treated with type I collagen. Under acidic conditions, type I collagen molecules are adsorbed on the surface of a dish in the non-fibrous form (left panel). Under neutral conditions at $37^{\circ} \mathrm{C}$, type I collagen molecules are reassembled into fibrils and adsorbed on the surface of dishes in the gel form (right panel). Please click here to view a larger version of this figure.

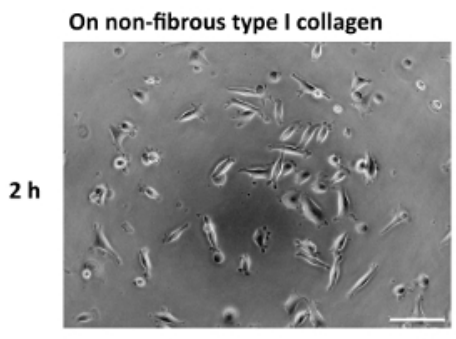

On type I collagen fibrils
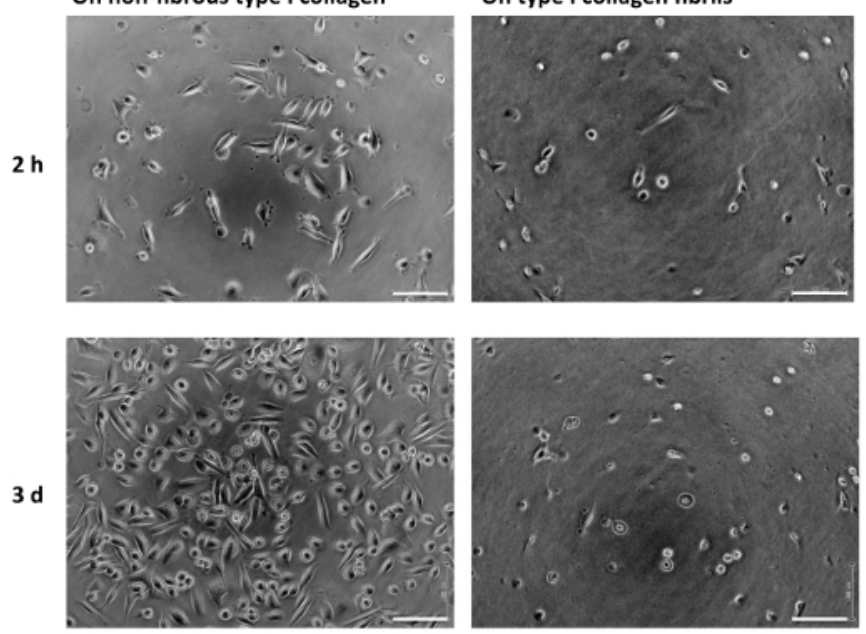

Figure 2: Morphology of FEPE1L-8 cells. FEPE1L-8 cells in K110 were cultured using the non-fibrous form (10 $\mu \mathrm{g} / \mathrm{mL}$; left panels) or fibril form ( $1 \mathrm{mg} / \mathrm{mL}$; right panels) of type I collagen for $2 \mathrm{~h}$ (upper panels) or 3 days (lower panels). White bars indicate $100 \mu \mathrm{m}$. Figure 2 has been modified from Fujisaki et al. in Figure 1E- $\mathbf{H}^{11}$. Please click here to view a larger version of this figure. 


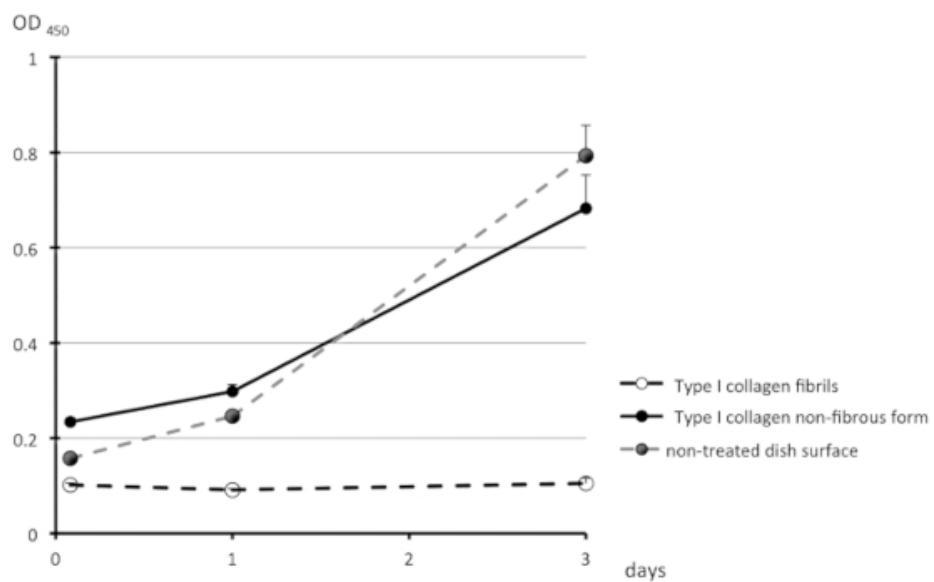

Figure 3: Proliferation of FEPE1L-8 cell. The number of viable cells were estimated on the non-fibrous form (black solid line, black filled circles) or on the fibril form (dotted line, open circle) of type I collagen, or untreated dish surfaces (gray dotted line, gray filled circles) for $2 \mathrm{~h}, 1$ day, and 3 days. Experiments were performed in triplicates and values are shown as means + SD. This figure has been modified from Fujisaki et al. in Figure $1 \mathbf{J}^{11}$. Please click here to view a larger version of this figure.

\section{Discussion}

Some ECM components, including type I collagen, form three-dimensional structures in vivo ${ }^{1}$. Culturing on such a three-dimensional, gel substrate provides more physiological conditions in vitro than on a two-dimensional, plastic surface $e^{1,2,3,4}$. Numerous protocols regarding the gel culture method have been reported, such as using type I collagen ${ }^{1,2,3,4,6,7,11}$, type IV collagen ${ }^{14,15}$, and Matrigel ${ }^{16}$. Type I collagen is a welldefined and widely used material because of its abundance and ease of handling. The features of purified type I collagen depend on the animal species, age, and purification methods ${ }^{5,17}$. Type I collagen can be purified using acetic acid and/or proteases, such as pepsin, papain, and proctase $^{5,17}$. Acid-soluble collagen maintains amino-telopeptides and protease-soluble collagen are cleaved amino-telopeptides ${ }^{5,17}$. The reserved length of amino-telopeptides depends on the type of proteases, and the presence of telopeptides affects fibril morphology and gel strength ${ }^{5,17}$. The viscosity of acid-soluble collagen fibrils is greater than that of protease-treated collagens ${ }^{17}$. In this study, we used acid-soluble bovine type I collagen. Pepsin-solubilized collagen can also be used in this gel culture protocol; however, the gel strength is weaker ${ }^{17}$. These differences in materials can cause cell behavioral differences, but they are currently not well understood.

The gel culture protocol described in this study is very simple. Many modifications of this method have been reported. One possible modification for the culture of keratinocytes is to mimic the basement membrane. Type IV collagen gels may be better to keep a basement membrane-like substrate structure in vitro ${ }^{15,18}$. However, a long incubation period is required for the preparation of type IV collagen gels ${ }^{14,15,18}$. Instead, mixing type IV collagen with type I collagen gels can produce novel culture substrates (type I/type IV collagen hybrid gels) ${ }^{19}$. These hybrid gels are easy to handle, require a short time for gelation, and yield more basement membrane-like conditions for keratinocytes. On type I/type IV collagen hybrid gels, keratinocytes survive, form colonies, and induce terminal differentiation ${ }^{19}$. This hybrid method has versatile applications.

On-gel culture using type I collagen affects cancer cells. On the fibril form of type I collagen, Akt activation and growth of Caco-2 cells (a colon cancer cell line) are suppressed ${ }^{7}$. In addition, the growth of human melanoma cells (M24met) on the fibril form is arrested at the G1/S checkpoint $^{20}$. Moreover, markedly increased levels of reactive oxygen species are observed in murine 3T3-L1 preadipocytes cultured on the fibril form. Furthermore, cell proliferation and migration are stimulated in opposite directions by the non-fibrous and fibril forms of type I collagen ${ }^{21}$.

\section{Disclosures}

The authors declare that they have no competing financial interests.

\section{Acknowledgments}

We are grateful to Dr. K. Sekiguchi (Institute for Protein Research, Osaka University), Dr. M. Yamada (Institute for Protein Research, Osaka University), Dr. T. Ikejima (China-Japan Research Institute of Medical and Pharmaceutical Sciences, Wuya College of Innovation, Shenyang Pharmaceutical University) and T. Hayashi (China-Japan Research Institute of Medical and Pharmaceutical Sciences, Wuya College of Innovation, Shenyang Pharmaceutical University) for helpful comments.

\section{References}

1. Kanta, J. Collagen matrix as a tool in studying fibroblastic cell behavior. Cell Adhesion \& Migration. 9 (4), $308-316$ (2015).

2. Kleinman, H. K. Fibroblast adhesion to collagen substrates. Methods of Enzymology. 82, 503-508 (1982).

3. Kleinman, H. K., et al. Use of extracellular matrix and its components in culture. In "Extracellular Matrix: A Practical Approach" (M. A. Haralson and J. R. Hassell, Eds., 1st ed., IRL Press, Oxford) 289-301 (1995). 
4. Yamato, M., Hayashi, T. Topological distribution of collagen binding sites on fibroblasts cultured within collagen gels.. In "Extracellular MatrixCell Interaction" (Y. Ninomiya, B. R. Olsen, and T. Ooyama, Eds.) 123-140 (1998).

5. Suzuki, Y., Someki, I., Adachi, E., Irie, S., Hattori, S. Interaction of collagen molecules from the aspect of fibril formation: Acid-soluble, alkalitreated and MMP1-digested fragments of type I collagen. Journal of Biochemistry. 126, 54-67 (1999).

6. Fujisaki, H., Hattori, S. Keratinocyte apoptosis on type I collagen gel caused by lack of laminin 5/10/11 deposition and Akt signaling. Experimental Cell Research. 280, 255-269 (2002).

7. Sasaki, J., Fujisaki, H., Adachi, E., Irie, S., Hattori, S. Delay of cell cycle progression and induction of death of cancer cells on type I collagen fibrils. Connective Tissue Research. 52 (3), 167-177 (2011).

8. Wang, Z., Li, R., Zhong, R. Extracellular matrix promotes proliferation, migration and adhesion of airway smooth muscle cells in a rat model of chronic obstructive pulmonary disease via upregulation of the PI3K/AKT signaling pathway. Molecular Medicine Reports. 18, 3143-3152 (2018).

9. Shih, Y.-R., V., Tseng, K.-F., Lai, H.-Y., Lin, C.-H., Lee, O. K. Matrix stiffness regulation of integrin\#mediated mechanotransduction during osteogenic differentiation of human mesenchymal stem cells. Journal of Bone and Mineral Research. 26, 730-738 (2011).

10. Breitkreutz, D., Koxholt, I., Thiemann, K., Nischt, R. Skin basement membrane: The foundation of epidermal integrity- BM functions and diverse roles of binding molecules nidogen and perlecan. BioMed Resesrch International. 179784 (2013).

11. Fujisaki, H., et al. Respective optimal calcium concentrations for proliferation on type I collagen fibrils in two keratinocyte line cells, HaCaT and FEPE1L-8. Regenerative Therapy. 8, 73-79 (2018).

12. Kaur P., McDougall J.K., Cone, R. Immortalization of primary human epithelial cells by cloned cervical carcinoma DNA containing human papillomavirus type 16 E6/E7 open reading frames. Journal of General Virology. 70, 1261-66 (1989).

13. Kaur, P., Carter, W.G. Integrin expression and differentiation in transformed human epidermal cells is regulated by fibroblasts. Journal of Cell Science. 103, 755-63 (1992).

14. Fujisaki, H., Adachi, E., Hattori, S. Keratinocyte differentiation and proliferation are regulated by adhesion to the three-dimensional meshwork structure of type IV collagen. Connective Tissue Research. 49 (6), 426-436 (2008).

15. Hirose, M., Kosugi, H., Nakazato, K., Hayashi, T. Restoration to a quiescent and contractile phenotype from a proliferative phenotype of myofibroblast-like human aortic smooth muscle cells by culture on type IV collagen gels. Journal of Biochemistry. 125, 991-1000 (1999).

16. Yonemura, S. Differential Sensitivity of Epithelial Cells to Extracellular Matrix in Polarity Establishment. Plos One. 9, e112922 (2014).

17. Sato, K., et al. Possible Involvement of Aminotelopeptide in Self-assembly and Thermal Stability of Collagen I as Revealed by Its Removal with Proteases. Journal of Biological Chemistry. 275 (33), 25870-25875 (2000).

18. Nakazato, K., Muraoka, M., Adachi, E., Hayashi, T. Gelation of lens capsule type IV collagen solution at a neutral pH. Journal of Biochemistry. 120, 889-894 (1996)

19. Hattori, S., et al. Type I -Type IV collagen hybrid gel European patent 2489375 (11F019-EP) Applicant: Japan Institute of leather Research and Osaka University. (2015).

20. Henriet, P., Zhong, Z.D., Brooks, P.C., Weinberg, K.I., DeClerck, Y.A. Contact with fibrillar collagen inhibits melanoma cell proliferation by upregulating p27KIP1. Proceedings of the National Academy of Sciences of the United States of America. 97 (18), 10026-31 (2000).

21. Liu, X., et al. Differential levels of reactive oxygen species in murine preadipocyte 3T3-L1 cells cultured on type I collagen molecule-coated and gel-covered dishes exert opposite effects on NF-KB-mediated proliferation and migration. Free Radical Research. 28, 1-16 (2018). 